\title{
AIDING AND ABETTING
}


This page intentionally left blank 


\section{AIDING AND ABETTING}

U.S. FOREIGN ASSISTANCE

AND STATE VIOLENCE

Jessica Trisko Darden

STANFORD UNIVERSITY PRESS

Stanford, California 
Stanford University Press

Stanford, California

(C) 2020 by the Board of Trustees of the Leland Stanford Junior University.

All rights reserved.

No part of this book may be reproduced or transmitted in any form or by any means, electronic or mechanical, including photocopying and recording, or in any information storage or retrieval system without the prior written permission of Stanford University Press.

Printed in the United States of America on acid-free, archival-quality paper

Library of Congress Cataloging-in-Publication Data

Names: Trisko Darden, Jessica, author.

Title: Aiding and abetting : U.S. foreign assistance and state violence / Jessica Trisko Darden.

Description: Stanford, California : Stanford University Press, 2020. | Includes bibliographical references and index.

Identifiers: LCCN 2019019672 (print) | LCCN 2019022027 (ebook) | ISBN 97815036IIo09 (electronic) | ISBN 978150361023I (cloth :alk. paper) | ISBN 9781503610996(pbk. :alk. paper)

Subjects: LCSH: Human rights-Developing countries. | Political persecution-Developing countries. | State-sponsored terrorismDeveloping countries. | Military assistance, American—Developing countries. | Economic assistance, American-Developing countries.

Classification: LCC JC599.D44 (ebook) | LCC JC599.D44 T75 2020 (print) | DDC $323 / .044-\mathrm{dc} 23$

LC record available at https://lccn.loc.gov/2019019672

Cover design: Olivier Ballou

Typeset by Westchester Publishing Services in Garamond Pro. 